\title{
Photoinduced Bidirectional Magnetism against Monodirectional Electronics in Square-Antiprismatic Octacyanometalates
}

\author{
Jun Ohara and Shoji Yamamoto* \\ Department of Physics, Hokkaido University, Sapporo 060-0810, Japan
}

\begin{abstract}
Irradiating $\mathrm{Cu}_{2} \mathrm{Mo}(\mathrm{CN})_{8} \cdot 8 \mathrm{H}_{2} \mathrm{O}$ with blue light induces a global magnetization, whereas succeeding irradiations with red or longer-wavelength light demagnetize this material. We solve the time-dependent Schrödinger equation for an extended Hubbard model to reproduce the photoreversible magnetism. Monitoring the photoinduced optical-conductivity and angle-resolved-photoemission spectra, we reveal that the magnetic round trip by way of ferromagnetism is far from a return in terms of electronics. While visible-light-induced magnetization has never been observed in the tungsten analog $\mathrm{Cu}_{2} \mathrm{~W}(\mathrm{CN})_{8} \cdot 5 \mathrm{H}_{2} \mathrm{O}$, infrared-light irradiation may magnetize this material as well.
\end{abstract}

Multifunctional magnetic materials based on octacyanometalates $^{1,2)}$ have been attracting considerable attention. In comparison with popular hexacyanometalates including Prussian blue analogs ${ }^{3,4)}$ well known to be spin-crossover complexes, higher-than-six-coordinate metal complexes can have various magnetic exchange pathways. $\left.{ }^{5,6}\right)$ The octacyano transition-metal ions $\left[M(\mathrm{CN})_{8}\right]^{3-/ 4-}(M=\mathrm{Mo}$, W) can take spatial configurations such as square antiprism (SAPR) of $\mathbf{D}_{4 d}$ point symmetry [Fig. 1(a)], dodecahedron (DD) of $\mathbf{D}_{2 d}$ point symmetry [Fig. 1(b)], and bicapped trigonal prism (BTP) of $\mathbf{C}_{2 v}$ point symmetry [Fig. 1(c)]. ${ }^{7-9)}$ Among others, the coordination networks comprising the square-antiprismatic anions $\left[\mathrm{Mo}^{\mathrm{IV}}(\mathrm{CN})_{8}\right]^{4-}$ and cations $\mathrm{Cu}^{2+}$ are so interesting as to exhibit photoswitchable magnetism. ${ }^{10-12)}$ Blue-light irradiation of the dicopper octacyanomolybdate $\mathrm{Cu}_{2} \mathrm{Mo}(\mathrm{CN})_{8} \cdot 8 \mathrm{H}_{2} \mathrm{O}$, abbreviated as $\mathrm{Cu}-\mathrm{Mo}$, at sufficiently low temperatures induces ferromagnetism, whereas subsequent longer-wavelength-light irradiation demagnetizes this material. ${ }^{13)}$ However, little is known about the photoexcited electronic state from the experimental viewpoint and we are almost ignorant of the photoinduced demagnetization as well as magnetization mechanisms.

In the $I 4 / m$ crystal structure of $\mathbf{C}_{4 h}$ point symmetry, ${ }^{14)}$ the constituent $\mathrm{Mo}^{\mathrm{IV}}$ and $\mathrm{Cu}^{\mathrm{II}}$ ions contribute their doubly occupied $4 d_{z^{2}}$ and singly occupied $3 d_{z x / y z}$ orbitals to the itinerant magnetism, respectively. The $3 d_{z x}\left(3 d_{y z}\right)$ orbitals are sitting on the $\mathrm{Cu}$ ions located at the vertices $1,2,5$, and $6(3,4,7$, and 8) of SAPR [Fig. 1(a)]. ${ }^{15)}$ We employ a multiband extended Hubbard model ${ }^{16-18)}$ to visualize the photoexcitation mechanism theoretically and encourage further explorations of related materials. The Hubbard Hamiltonian is treated within a Hartree-Fock (HF) scheme, and it is revealed that the mixedvalent states shown in Fig. 2 compete with each other at sufficiently low temperatures. ${ }^{19)}$ The paramagnetic (PM) state can take the valence arrangement $\mathrm{Cu}^{\mathrm{II}}-\mathrm{Mo}^{\mathrm{IV}}-\mathrm{Cu}^{\mathrm{II}}$ and appears as the ground state unless the electronic correlation is not suffi-

*yamamoto@phys.sci.hokudai.ac.jp (a) SAPR

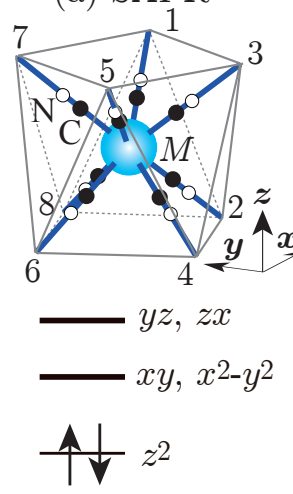

(b) DD

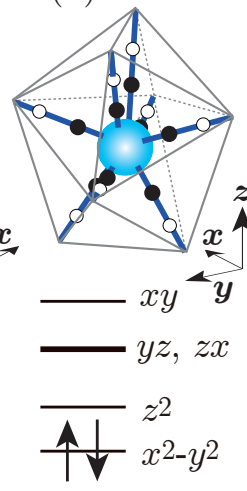

(c) BTP

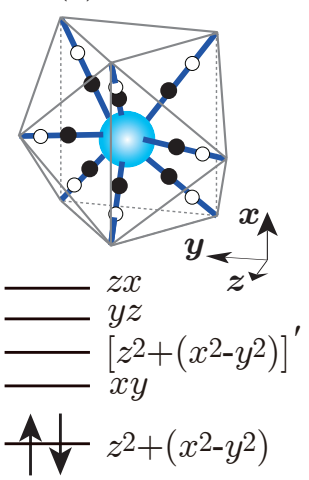

Fig. 1. (Color online) Spatial configurations of $\left[M^{\mathrm{IV}}(\mathrm{CN})_{8}\right]^{4-}(M=\mathrm{Mo}$ W): (a) Square antiprism (SAPR), (b) dodecahedron (DD), and (c) bicapped trigonal prism (BTP). The crystal-field splittings of the $4 d(5 d)$ orbitals on the Mo $(\mathrm{W})$ site and the electron configurations are also shown, where thick lines denote doubly degenerate levels. (a) PM

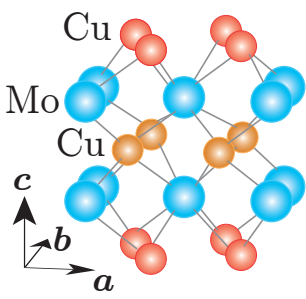

(b) FM

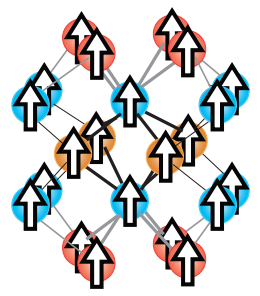

(c) $\mathrm{CDP}$

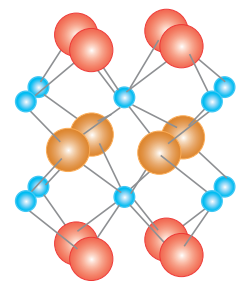

Fig. 2. (Color online) (a) Paramagnetic (PM), (b) ferromagnetic (FM), and (c) charge-disproportionated (CDP) states in the dicopper octacyanomolybdate $(\mathrm{Cu}-\mathrm{Mo})$, where varying circles and arrows describe varying electron and spin densities, respectively. The crystal axes are denoted by $\boldsymbol{a}, \boldsymbol{b}$, and $\boldsymbol{c}$.

ciently strong. In a strong correlation regime, the ferromagnetic (FM) state having a global magnetization is stable. This state shows a spatially uniform electron distribution and exhibits metallic behavior. A non-magnetized solution different from the PM state, the charge-disproportionated (CDP) 
state, appears in $\mathrm{Cu}-\mathrm{Mo}$. The CDP state takes the valence arrangement $\mathrm{Cu}^{\mathrm{I}}-\mathrm{Mo}^{\mathrm{VI}}-\mathrm{Cu}^{\mathrm{I}}$ (fully-filled $\mathrm{Cu}$ and vacant $\mathrm{Mo}$ sites) and plays an important role in the photoexcited $\mathrm{Cu}-\mathrm{Mo}$. The model parameters are tuned so as to reproduce the valence arrangement $\mathrm{Cu}^{\mathrm{II}}-\mathrm{Mo}^{\mathrm{IV}}-\mathrm{Cu}^{\mathrm{II}}$ in the $\mathrm{PM}$ ground state. ${ }^{18)}$

We consider Dzyaloshinskii-Moriya (DM) and Zeeman interactions in a time-evolutional calculation. The DM interaction arises from the crystalline structure, ${ }^{20-22)}$ which allows the total magnetization to fluctuate. We apply a weak external magnetic field along the $c(z)$ axis in line with the experimental setup. ${ }^{13)}$ Though the Zeeman interaction ${ }^{23)}$ serves to lift the spin up-down degeneracy, the applied field is sufficiently weak and can never justify in itself the observed giant photomagnetization. We introduce the vector potential $\boldsymbol{A}(t)$ to photoirradiate the system, i.e., multiply the electron hopping term by the Peierls phase factor $\exp \left[-\frac{\mathrm{i} e}{\hbar v} \boldsymbol{A}(t) \cdot \delta\right],{ }^{24)}$ where $e$, $v$, and $\delta$ are the elementary charge, speed of light, and relative vector between cyano-bridged metals, respectively. Time evolution of an electronic wavefunction is obtained by integrating the time-dependent Schrödinger equation $i \hbar|\dot{\Psi}(t)\rangle=$ $\mathcal{H}_{\mathrm{HF}}(t)|\Psi(t)\rangle$, where $\mathcal{H}_{\mathrm{HF}}(t)$ is the total Hamiltonian treated within the HF scheme. ${ }^{25,26)}$ At $t \rightarrow-\infty$, the wavefunction $|\Psi(t)\rangle$ corresponds to the initial PM state. Hereafter, the transfer integral between the nearest neighboring cyano-bridged metals $t_{\mathrm{MoCu}}$ is set to $0.65 \mathrm{eV}$, which is comparable to the transfer integral in typical Prussian blue analogs. ${ }^{27,28)}$

Figure 3(a) shows the magnetization dynamics in $\mathrm{Cu}-\mathrm{Mo}$ induced by continuous laser lights polarized parallel to $\boldsymbol{a}$ axis,

$$
\begin{aligned}
\boldsymbol{A}(t)=\sum_{i=1}^{4} & \boldsymbol{A}_{i} \frac{\left[1+\tanh \left\{\gamma\left(t-t_{i}^{\mathrm{on}}\right)\right\}\right]}{2} \frac{\left[1+\tanh \left\{-\gamma\left(t-t_{i}^{\mathrm{off}}\right)\right\}\right]}{2} \\
& \times \cos \omega_{i} t
\end{aligned}
$$

with various frequencies $\omega_{i}$ of duration $\tau_{i} \equiv t_{i}^{\text {off }}-t_{i}^{\text {on }}$. The power and duration of the laser lights are on the order of $10^{12} \mathrm{~J} \mathrm{~s}^{-1} \mathrm{~cm}^{-2}$ and $10^{-12} \mathrm{~s}$, respectively. They are standard in photoinduced-dynamics calculations ${ }^{29)}$ but not necessarily comparable with experimental observations. ${ }^{13)}$ So, we simulate and reproduce any experiment based on the average energy absorbed by each unit cell during the light irradiation. We assume that effects of energy dissipation are negligible in the magnetization dynamics. ${ }^{11,30)}$ The irradiation with $\hbar \omega=2.6 \mathrm{eV}$ light induces a global magnetization, whereas the subsequent irradiation with $\hbar \omega=1.9 \mathrm{eV}$ light conversely demagnetizes the system. The magnetization further decreases gradually upon the third and fourth irradiations with longer-wavelength light. The simulations well reproduce the visible-light-induced magnetization and demagnetization dynamics in the experimental observation. ${ }^{13)}$ Details of the electronic structures are discussed later. We further investigate photoinduced dynamics in the tungsten analog complex $\mathrm{Cu}_{2} \mathrm{~W}(\mathrm{CN})_{8} \cdot 5 \mathrm{H}_{2} \mathrm{O},{ }^{10)}$ abbreviated as $\mathrm{Cu}-\mathrm{W}$. Its ground state shows the valence arrangement $\mathrm{Cu}^{\mathrm{II}}-\mathrm{W}^{\mathrm{IV}}-\mathrm{Cu}^{\mathrm{II}}$, where the $\mathrm{W}^{\mathrm{IV}}$ ions contribute their doubly occupied $5 d_{z^{2}}$ orbitals [Fig. 1(a)] to the physical properties. This situation is qualitatively the
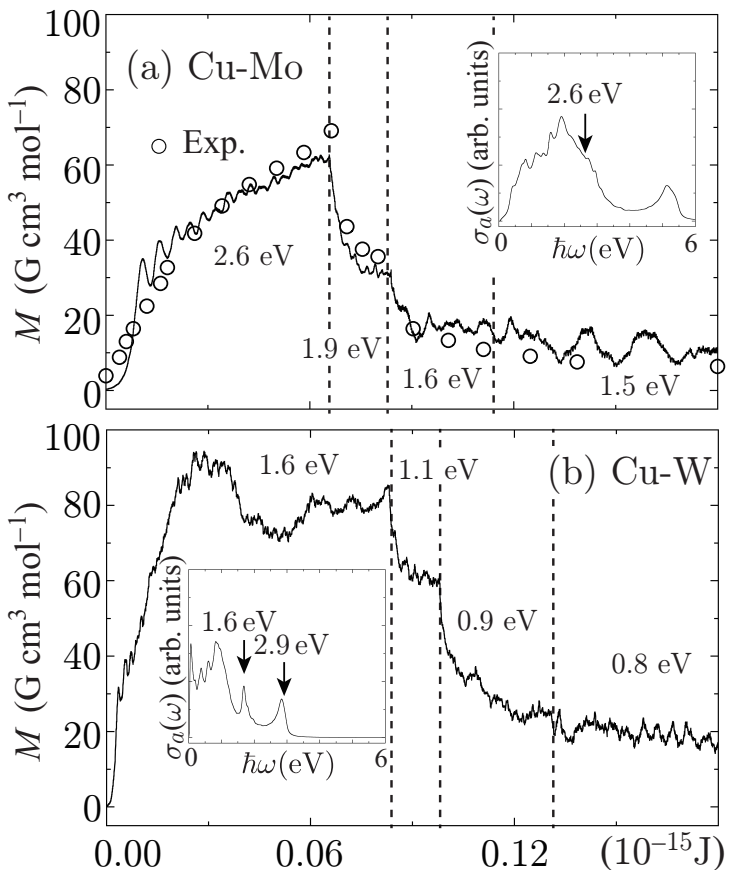

Fig. 3. (Color online) Magnetization in the square-antiprismatic octacyanometalates due to successive photoirradiation plotted as a function of the average energy absorbed by each unit cell, calculations (solid lines) and experiments (open circles). ${ }^{13)}$ (a) $\mathrm{Cu}-\mathrm{Mo}$ : the photon energy, duration, and amplitude are taken as $\hbar \omega_{i}=2.6,1.9,1.6,1.5 \mathrm{eV}, \tau_{i}=0.9,0.3,0.3,0.5 \mathrm{ps}$, and $\left|\boldsymbol{A}_{i}\right| e a / \hbar v=0.5,0.6,1.0,1.1$, respectively. The switching time $2 \gamma^{-1}$ is set to 0.03 ps. (b) $\mathrm{Cu}-\mathrm{W}$ : the same as (a) but $\hbar \omega_{i}=1.6,1.1,0.9,0.8 \mathrm{eV}$. Insets in (a) and (b) show the polarized (\|a) optical conductivities in the ground state.

same as the PM state in Cu-Mo. However, irradiations of the ground state with $\hbar \omega=2.5$ and $3.0 \mathrm{eV}$ lights induce no significant magnetization. ${ }^{10)}$ According to these observations, $\mathrm{Cu}$ $\mathrm{W}$ seems not to be a photomagnet. We set the transfer integral $t_{\mathrm{WCu}}$ to $0.42 \mathrm{eV}\left(<t_{\mathrm{MoCu}}\right)$ by considering metal-substitution effects $^{31)}$ and irradiate the ground state with the continuous laser light. The violet-light $(\hbar \omega=2.9 \mathrm{eV})$ irradiation induces no global magnetization, but the sufficiently low-energy-light ( $\hbar \omega=1.6 \mathrm{eV}$ ) irradiation causes a PM-to-FM transition [Fig. 3(b)]. The magnetization induced by this infrared light disappears with succeeding irradiations. Thus, $\mathrm{Cu}-\mathrm{W}$, as well as $\mathrm{Cu}-\mathrm{Mo}$, shows photoswitchable magnetism. The insets in Fig. 3 show the ground-state optical conductivities along $\boldsymbol{a}$ axis. ${ }^{32-34)}$ A major absorption band of $\mathrm{Cu}-\mathrm{W}$ is observed in an infrared region, while that of $\mathrm{Cu}-\mathrm{Mo}$ is seen in a visible region. The electron-hole pair generation by these optical absorptions leads to the magnetic transitions. Note that these ground-state absorption spectra are also in good agreement with the observations. ${ }^{10,13)}$

Figure 4(a) shows the magnetization dynamics in $\mathrm{Cu}-\mathrm{Mo}$ induced by pulsed laser lights polarized parallel to $\boldsymbol{a}$ axis,

$$
\boldsymbol{A}(t)=\boldsymbol{A} \sum_{i=1}^{4} e^{-\gamma^{2}\left(t-t_{i}\right)^{2}} \cos \omega_{i} t
$$

with various frequencies $\omega_{i}$ of width $2 \gamma^{-1}$. In order to mon- 


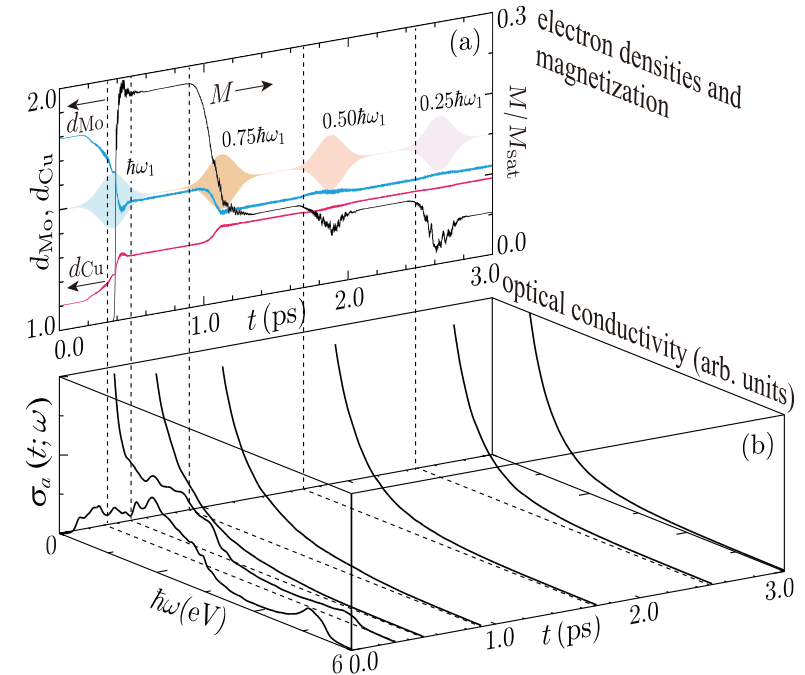

Fig. 4. (Color online) Photoinduced magnetization and demagnetization dynamics: (a) The averaged electron density on $\mathrm{Mo}(\mathrm{Cu})$ sites $d_{\mathrm{Mo}(\mathrm{Cu})}$ and the total magnetization divided by the saturation magnetization $M / M_{\mathrm{sat}}$. The pumping laser pulses are such that $\hbar \omega_{i}=2.6[1-0.25(i-1)] \mathrm{eV}$ and $t_{i}=0.4+0.75(i-1) \mathrm{ps}$, illustrated schematically in (a). The amplitude $|\boldsymbol{A}| e a / \hbar v$ and width $2 \gamma^{-1}$ of each pulse are set to 2.0 and $0.25 \mathrm{ps}$, respectively. (b) Photoinduced optical conductivities along $\boldsymbol{a}$ axis at several stages in the magnetization and demagnetization processes.

itor the magnetic dynamics in more detail, we calculate the electron densities on transition-metal sites and the total magnetization as functions of time. The irradiation with $\hbar \omega_{1}$ light firstly causes the electron transfer from Mo to $\mathrm{Cu}$ sites $(0.2$ $\sim 0.3 \mathrm{ps}$ ) and secondary induces the substantial magnetization $(0.4 \mathrm{ps})$. Since the spin degeneracy is lifted due to the magnetic field, the higher-lying down-spin electrons are selectively excited, ${ }^{35)}$ which leads to a net spin density on each site. After that, the DM interaction begins to work. The magnetization switching speed increases in proportion to the increase in the laser amplitude $|\boldsymbol{A}|{ }^{36)}$ In this FM transition, metallization proceeds the magnetization as is demonstrated by the optical conductivities in Fig. 4(b), where the Drude response is observed. The magnetization process reads as an insulator-to-metal transition. The subsequent irradiation with $0.75 \hbar \omega_{1}$ light demagnetizes the system $(0.9 \sim 1.3 \mathrm{ps})$, while electrons are further transferred from $\mathrm{Mo}$ to $\mathrm{Cu}$ sites and the conductivity does not change. This is not just a reverse process of the photomagnetization. When the system is further irradiated with $0.50 \hbar \omega_{1}$ and $0.25 \hbar \omega_{1}$ lights successively, the magnetization gets small step by step. ${ }^{37)}$ The transferred electrons never turn back to Mo sites and the system remains metallic. The final demagnetized state is totally different from the initial insulating PM state. Qualitatively the same phase transitions occur when the system is irradiated with the continuous laser lights (Fig. 3). Thus, the magnetic round trip is far from a return in terms of the electric conductivity.

Figure 5(a) shows the one-particle excitation spectrum $D(t ; \boldsymbol{k}, \omega)$ corresponding to an angle-resolved photoemission spectroscopy (ARPES) spectrum: ${ }^{38)} D(t ; \boldsymbol{k}, \omega)=H(t ; \boldsymbol{k}, \omega)+$

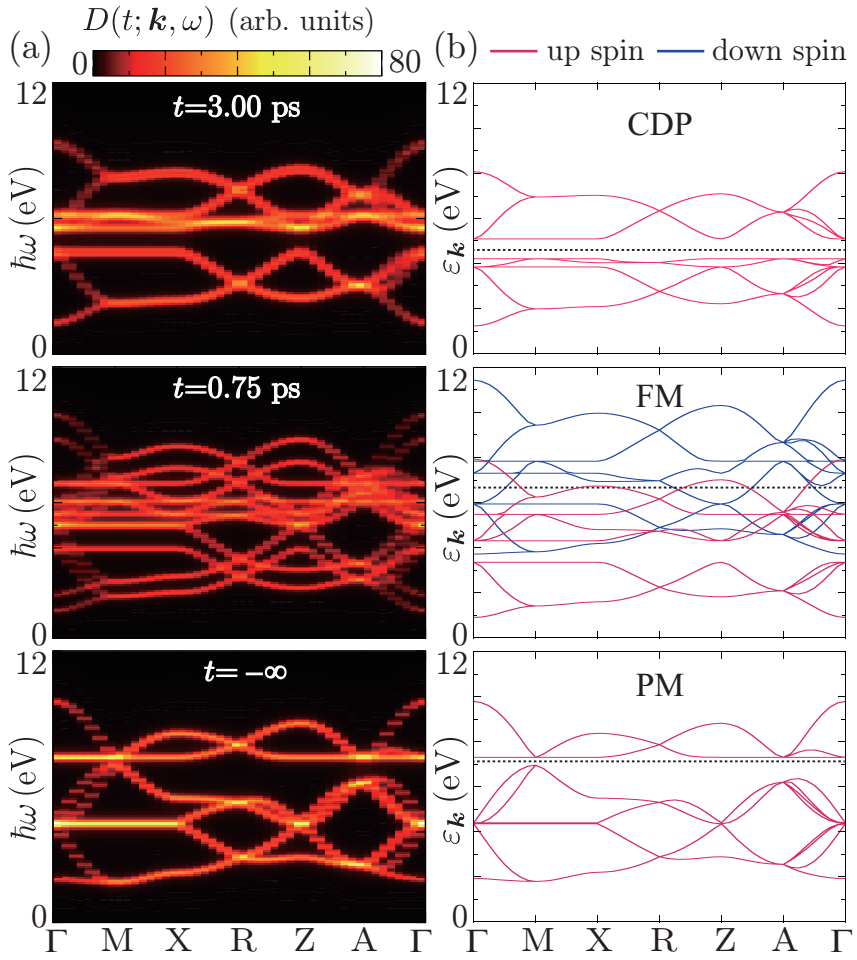

Fig. 5. (Color online) (a) ARPES spectra of the initial PM $(t=-\infty)$, photomagnetized ( $t=0.75 \mathrm{ps}$ ), and final demagnetized ( $t=3.0 \mathrm{ps})$ states. (b) Dispersion relations of up- and down-spin electrons in the PM, FM, and CDP ground states, where a dotted line in each panel denotes the Fermi level.

$E(t ; \boldsymbol{k}, \omega)$ with

$$
\begin{aligned}
& E(t ; \boldsymbol{k}, \omega)=\sum_{\boldsymbol{k}, \chi} \delta\left(\hbar \omega-\varepsilon_{\boldsymbol{k}, \chi}\right)\left\langle\Psi(t)\left|\tilde{c}_{\boldsymbol{k}, \chi}^{\dagger} \tilde{c}_{\boldsymbol{k}, \chi}\right| \Psi(t)\right\rangle, \\
& H(t ; \boldsymbol{k}, \omega)=\sum_{\boldsymbol{k}, \chi} \delta\left(\hbar \omega-\varepsilon_{\boldsymbol{k}, \chi}\right)\left\langle\Psi(t)\left|\tilde{c}_{\boldsymbol{k}, \chi} \tilde{c}_{\boldsymbol{k}, \chi}^{\dagger}\right| \Psi(t)\right\rangle,
\end{aligned}
$$

where $\varepsilon_{\boldsymbol{k}, \chi}$ is a HF-level energy of $\mathcal{H}_{\mathrm{HF}}(t)$ and $\tilde{c}_{\boldsymbol{k}, \chi}^{\dagger}$ creates the electron labeled by the momentum $\boldsymbol{k}$ and the band in$\operatorname{dex} \chi(=1 \sim 24)$. We compare band structures of the photoexcited system with those of the PM, FM, and CDP ground states [Fig. 5(b)]. We can obtain each ground state by tuning the on-site Coulomb repulsion $U_{\mathrm{Cu} / \mathrm{Mo}}{ }^{19)}$ The first irradiation with $\hbar \omega=2.6 \mathrm{eV}$ light excites electrons around $\Gamma$ point. The spatially uniform oscillation leads to the appearance of the macroscopic magnetization. In the photomagnetized metallic state $(t=0.75 \mathrm{ps}$ ), the spin degeneracy is totally lifted just like the itinerant FM ground state. In the photoexcited state, the spin index up or down is no longer a good quantum index due to the DM interaction. Further photoirradiation gives rise to the charge-transfer excitations accompanied by spin flips, which leads to the reduction in the net spin density on each site. It is observed as convergence of the split bands. The band structure of the thus demagnetized state $(t=3.00$ ps) is very close to that of the CDP ground state. The static CDP structure is stable in the condition $U_{\mathrm{Mo}} \gg U_{\mathrm{Cu}},{ }^{19)}$ but this situation is hardly realized because Mo $4 d$ orbitals are 
more diffuse than $\mathrm{Cu} 3 d$ ones in general. Such a hidden structure appears in the photoexcited $\mathrm{Cu}-\mathrm{Mo}$ and the final steady state should be called the nonequilibrium CDP state. Note that further light irradiation of excited states leads to emission as well as absorption of photons. Photoexcited electrons partially turn back to the valence bands. Since a resonant energy varies with changes in the band structure [Fig. 5(a)], emergence of the bidirectional magnetism requires the successive irradiation with varying wavelength light.

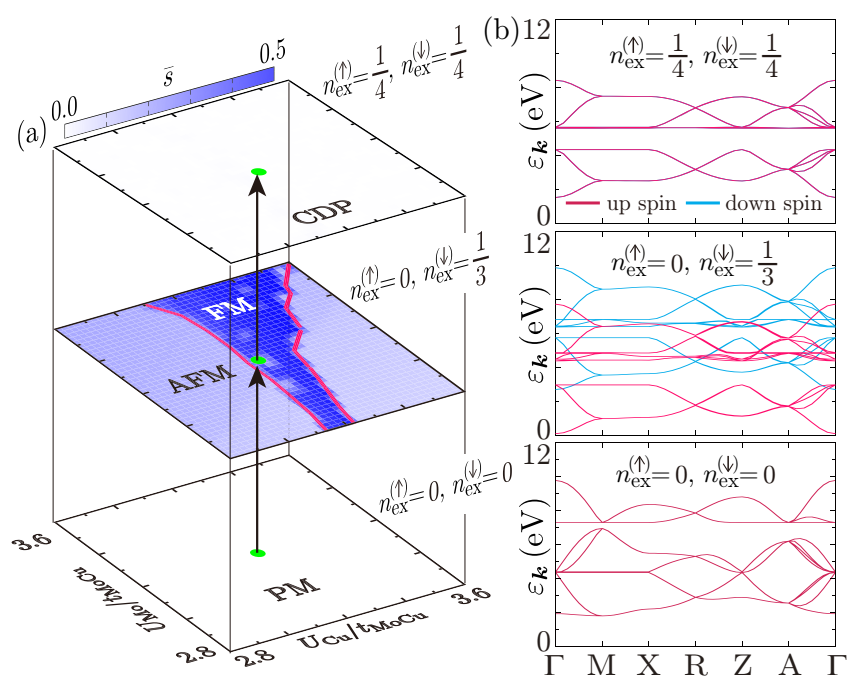

Fig. 6. (Color online) (a) Phase competition in the ground and photoexcited states on the $U_{\mathrm{Cu}}-U_{\mathrm{Mo}}$ plane, where the averaged spin density $\bar{s}$ (= $\left|s_{\mathrm{Mo}}\right| / 3+2\left|s_{\mathrm{Cu}}\right| / 3$ ) is also shown. (b) Band structures of the ground and photoexcited states at $U_{\mathrm{Mo}}=U_{\mathrm{Cu}}=3.2 t_{\mathrm{MoCu}}$.

Figure 6(a) shows the phase competition in the ground and excited states. We tune the electron population of the bands to simulate the photoexcitation, where the population is measured by numbers of excited up-spin $(\uparrow)$ and down-spin $(\downarrow)$ electrons per site, $n_{\mathrm{ex}}^{(\uparrow)}$ and $n_{\mathrm{ex}}^{(\downarrow)}$. As is shown in Fig. 6(a), we make phase diagrams of the static Hubbard Hamiltonian under several conditions. We focus on the region around the present Coulomb parameters $U_{\mathrm{Mo}}=U_{\mathrm{Cu}}=3.2 t_{\mathrm{MoCu}}$, in which the PM ground state predominates. For example, the condition $n_{\mathrm{ex}}^{(\uparrow)}=0$ and $n_{\mathrm{ex}}^{(\downarrow)}=1 / 3$ means that all of the up-spin electrons are in valence bands but half of the down-spin ones in valence bands are excited to conduction bands. On the basis of these electron distributions, we calculate order parameters, such as electron and spin densities, and then obtain the mean-field Hamiltonian describing photoexcited states. Diagonalizing this Hamiltonian, we find an energy and band structure of the excited state. We simulate the first photoexcitation by considering the condition $n_{\mathrm{ex}}^{(\uparrow)}=0$ and $n_{\mathrm{ex}}^{(\downarrow)}=1 / 3$, which describes the spin-selective electron excitation. The FM and antiferromagnetic (AFM) ordering between the spins on $\mathrm{Mo}$ and $\mathrm{Cu}$ sites compete with each other. At the present parameter point, the FM structure appears with the electronhole pair excitations. Considering the spin mixing and photon
Table I. Theoretical evaluation of whether or how photoswitchable magnetism occurs in various square-antiprismatic octacyanometalates.

\begin{tabular}{cccc}
\hline \hline $\begin{array}{c}\text { Constituent } \\
\text { metals }\end{array}$ & $\begin{array}{c}\text { Electron } \\
\text { occupancy }\end{array}$ & $\begin{array}{c}\text { Pump light } \\
\text { energy }\end{array}$ & $\begin{array}{c}\text { Photoswitchable } \\
\text { magnetism }\end{array}$ \\
\hline $\mathrm{Cu}_{2}^{\mathrm{II}} \mathrm{Mo}^{\mathrm{IV}}$ & $3 d^{9} 4 d^{2}$ & $1.5 \sim 2.6 \mathrm{eV}$ & Yes \\
$\mathrm{Cu}_{2}^{\mathrm{II}} \mathrm{W}^{\mathrm{IV}}$ & $3 d^{9} 5 d^{2}$ & $0.8 \sim 1.6 \mathrm{eV}$ & Yes \\
$\mathrm{Ni}_{2} \mathrm{Mo}^{\mathrm{IV}}$ & $3 d^{8} 4 d^{2}$ & - & No \\
\hline \hline
\end{tabular}

emission upon further light irradiations, we take the condition $n_{\mathrm{ex}}^{(\uparrow)}=n_{\mathrm{ex}}^{(\downarrow)}=1 / 4$ as the final steady state. The CDP structure becomes more stable than the PM one under this nonequilibrium situation. Figure 6(b) shows the band structure under each condition. Its $n_{\mathrm{ex}}^{(\sigma)}(\sigma=\uparrow, \downarrow)$ dependence is reminiscent of the time evolution of the photoexcited state shown in Fig. 5(a). Thus, the bidirectional photoswitching of magnetization reads as the multistep photoinduced phase transition.

Cobalt octacyanotungstates $\mathrm{Co}^{\mathrm{III}}-\mathrm{W}^{\mathrm{IV}}$ in the DD [Fig. 1(b)] and BTP [Fig. 1(c)] geometries also exhibit photoinduced ferromagnetism. ${ }^{39,40)}$ The $\mathrm{Co}^{\mathrm{III}}$ and $\mathrm{W}^{\mathrm{IV}}$ ions in the ground state contribute their vacant $3 d_{z^{2}}$ and fully-filled $5 d_{\left(x^{2}-y^{2}\right) /\left[z^{2}+\left(x^{2}-y^{2}\right)\right]}$ orbitals to the photomagnetism. ${ }^{39-41)}$ Unlike the PM ground states in $\mathrm{Cu}^{\mathrm{II}}-\mathrm{Mo}^{\mathrm{IV}}$ and $\mathrm{Cu}^{\mathrm{II}}-\mathrm{W}^{\mathrm{IV}}$, the Coulomb repulsion between $\mathrm{Co}$ and $\mathrm{W}$ sites stabilizes the valence arrangement $\mathrm{Co}^{\mathrm{III}}-\mathrm{W}^{\mathrm{IV}}$. The observed magnetization is about an order of magnitude larger than that in the SAPR systems. Four Co ions surround each $\mathrm{W}$ ion, i.e., half the $\mathrm{CN}$ groups of $\mathrm{W}(\mathrm{CN})_{8}$ are not linked to Co. Therefore, the $\mathrm{Co}-\mathrm{W}$ intermetallic Coulomb repulsion is thus weaker than the $\mathrm{Cu}-\mathrm{Mo}$ one in the SAPR geometry. The photodoped carriers are more itinerant in such a situation, leading to larger magnetization. The photomagnetism owes much to the coordination geometry.

The multistep photoinduced phase transitions in the dicopper octacyanometalates are not a turn-around process such as a bidirectional phase transition. ${ }^{42,43)}$ The visible- or infrared-light irradiation indeed induces a turn-around magnetic change, but it arises from one-way electronic transfer. The electron-hole pair excitation around $\Gamma$ point, which brings about a spatially uniform oscillation, causes the substantial increase of the global magnetization. Therefore, adjustment of the pump light energy is considerably important. The optical conductivity measurement in the low energy region below $1 \mathrm{eV}$ is needed to detect the one-way electronic transfer. We consider a band-filling dependence of the photoswitchable magnetism. Replacement of $3 d_{y z / z x}^{1}$ by $3 d_{y z / z x}^{0}$ changes the total band filling $v$ from $2 / 3$ to $1 / 3$. It corresponds to substitution of $\mathrm{Ni}$ for $\mathrm{Cu}^{44)}$ At $v=1 / 3$, electrons on anion sites feel no intermetallic Coulomb repulsion. Such a situation is much stabler than the PM state in $\mathrm{Cu}-\mathrm{Mo}$ or $\mathrm{Cu}-\mathrm{W}$. We confirmed that any photoirradiation induces neither global nor stable magnetization in the Ni-Mo system. As is shown in Table I, the choice of cations, as well as pumping energy, is decisive of photosensitivity of the system. Photomagnetism in DD and BTP complexes, their demagnetization mechanisms in partic- 
ular, are also an intriguing topic in the future.

The authors are grateful to S. Ohkoshi and H. Tokoro for valuable comments and discussion. This work was supported by the Ministry of Education, Culture, Sports, Science and Technology of Japan.

1) S. F. A. Kettle and R. V. Parish, Spectrochim. Acta 21, 1087 (1965).

2) O. Kalisky and M. Shirom, J. Photochem. 7, 215 (1977).

3) O. Sato, T. Iyoda, A. Fujishima, and K. Hashimoto, Science 272, 704 (1996).

4) M. Verdaguer, A. Bleuzen, V. Marvaud, J. Vaissermann, M. Sueleiman, C. Desplanches, A. Scuiller, C. Train, R. Garde, G. Gelly, C. Lomenech, I. Rosenman, P. Veillet, C. Cartier, and F. Villain, Coord. Chem. Rev. 190-192, 1023 (1999).

5) B. Sieklucka, R. Podgajny, P. Przychodzeń, and T. Korzeniak, Coord. Chem. Rev. 249, 2203 (2005)

6) P. Przychodzeń, T. Korzeniak, R. Podgajny, and B. Sieklucka, Coord. Chem. Rev. 250, 2234 (2006).

7) E. L. Muetterties and C. M. Wright, Q. Rev. Chem. Soc. 21, 109 (1967).

8) R. A. Pribush and R. D. Archer, Inorg. Chem. 13, 2556 (1974).

9) J. K. Burdett, R. Hoffmann, and R. C. Fay, Inorg. Chem. 17, 2553 (1978).

10) G. Rombaut, C. Mathonière, P. Guionneau, S. Golhen, L. Ouahab, M. Verelst, and P. Lecante, Inorg. Chim. Acta 326, 27 (2001).

11) S. Ohkoshi, N. Machida, Z. J. Zhong, and K. Hashimoto, Synth. Met. 122, 523 (2001)

12) X.-D. Ma, T. Yokoyama, T. Hozumi, K. Hashimoto, and S. Ohkoshi, Phys. Rev. B 72, 094107 (2005).

13) S. Ohkoshi, H. Tokoro, T. Hozumi, Y. Zhang, K. Hashimoto, C. Mathonière, I. Bord, G. Rombaut, M. Verelst, C. C. dit Moulin, and F. Villain, J. Am. Chem. Soc. 128, 270 (2006)

14) See Supplemental Material, where the detailed crystal structure of the square-antiprismatic octacyanometalates is presented in Section S1.

15) See Supplemental Material, where the arrangement of $4 d_{z^{2}}, 3 d_{z x}$, and $3 d_{y z}$ orbitals in the material is presented in Section S1.

16) J. Ohara and S. Yamamoto, Europhys. Lett. 87, 17006 (2009).

17) S. Yamamoto, J. Ohara, and M. Ozaki, J. Phys. Soc. Jpn. 79, 044709 (2010).

18) See Supplemental Material, where the model Hamiltonian and its parameters are presented in Sections S1 and S2, respectively.

19) See Supplemental Material, where the ground-state phase diagram is numerically obtained in Section S2.

20) J. Stein, O. Entin-Wohlman, and Amnon Aharony, Phys. Rev. B 53, 775 (1996).

21) B. Keimer, D. Casa, A. Ivanov, J. W. Lynn, M. v. Zimmermann, J. P. Hill, D. Gibbs, Y. Taguchi, and Y. Tokura, Phys. Rev. Lett. 85, 3946 (2000).

22) See Supplemental Material, where the detailed expression of the DM interaction and its effects on the photoexcited states are discussed in Section S3.

23) See Supplemental Material, where the detailed expression of the Zeeman interaction is presented in Section S3.

24) A. Terai and Y. Ono, Prog. Theor. Phys. Suppl. 113, 177 (1993).

25) Y. Tanaka and K. Yonemitsu, J. Phys. Soc. Jpn. 79, 024712 (2010).

26) S. Yamamoto, J. Phys. Soc. Jpn. 80, 084713 (2011).

27) T. Kawamoto, Y. Asai, and S. Abe, Phys. Rev. B 60, 12990 (1999).

28) K. Yoshizawa, F. Mohri, G. Nuspl, and T. Yamabe, J. Phys. Chem. B 102, 5432 (1998).

29) K. Yabana, T. Sugiyama, Y. Shinohara, T. Otobe, and G. F. Bertsch, Phys. Rev. B 85, 045134 (2012).

30) We investigated interplay of electronic and lattice degrees of freedom in this system by employing a Peierls-Hubbard Hamiltonian. No lattice deformation occurs in the PM, FM, CDP states and no energy dissipation from the electron to lattice systems is observed in any time-evolutional calculation.

31) See Supplemental Material, where the metal-substitution effects on the intermetallic transfer integral is discussed based on a perturbative consideration in Section S4.

32) J. Wagner, W. Hanke, and D. J. Scalapino, Phys. Rev. B 43, 10517 (1991).

33) D. J. Scalapino, S. R. White, and S. C. Zhang, Phys. Rev. Lett. 68, 2830 (1992).

34) See Supplemental Material, where the expressions of polarized optical conductivities consisting of the regular part and Drude component are presented in Section S5.

35) See Supplemental Material, where the spin-dependent electron excitation is discussed in more detail in Section S6.

36) See Supplemental Material, where the laser-amplitude dependence of the photoinduced magnetization dynamics and the effect of the magnetic field on the global magnetization are discussed in Section S6.

37) See Supplemental Material, where the Coulomb-repulsion and DMinteraction dependences of the photoswitchable magnetism are shown in Section S6.

38) Y. Kanamori, J. Ohara, and S. Ishihara, Phys. Rev. B 86, 045137 (2012).

39) N. Ozaki, H. Tokoro, Y. Hamada, A. Namai, T. Matsuda, S. Kaneko, and S. Ohkoshi, Adv. Funct. Mater. 22, 2089 (2012).

40) Y. Arimoto, S. Ohkoshi, Z. J. Zhong, H. Seino, Y. Mizobe, and K Hashimoto, J. Am. Chem. Soc. 125, 9240 (2003).

41) S. Ramakrishnan and E. D. Jemmis, J. Organomet. Chem. 865, 37 (2018).

42) S. Koshihara, Y. Tokura, K. Takeda, and T. Koda, Phys. Rev. B 52, 6265 (1995).

43) L. Zhang and S. Yamamoto, J. Phys. Soc. Jpn. 83, 064708 (2014).

44) H. Zhou, Y.-Y. Chen, A.-H. Yuan, and X.-P. Shen, Inorg. Chem. Commun. 11, 363 (2008). 


\title{
Supplemental Material for \\ "Photoinduced Bidirectional Magnetism against Monodirectional Electronics in Square-Antiprismatic Octacyanometalates"
}

\author{
Jun Ohara and Shoji Yamamoto*
}

Department of Physics, Hokkaido University, Sapporo 060-0810, Japan

\section{S1. Modeling}

To describe the supramolecular coordination network comprising $\mathrm{Mo}^{\mathrm{IV}}$ and $\mathrm{Cu}^{\mathrm{II}}$ ions [Fig. 1(a)], we employ a threeorbital extended Hubbard Hamiltonian, ${ }^{1,2)}$

$$
\begin{aligned}
& \mathcal{H}_{\mathrm{H}}=\sum_{\boldsymbol{n}, \sigma}\left[\sum_{i=1}^{8}\left(\varepsilon_{\mathrm{Cu}} n_{\boldsymbol{n}: \mathrm{Cu}(i) \sigma}+\frac{U_{\mathrm{Cu}}}{2} n_{\boldsymbol{n}: \mathrm{Cu}(i) \sigma} n_{\boldsymbol{n}: \mathrm{Cu}(i)-\sigma}\right)\right. \\
& \left.+\sum_{j=1}^{4}\left(\varepsilon_{\mathrm{Mo}} n_{n: \mathrm{Mo}(j) \sigma}+\frac{U_{\mathrm{Mo}}}{2} n_{n: \mathrm{Mo}(j) \sigma} n_{n: \mathrm{Mo}(j)-\sigma}\right)\right] \\
& +\sum_{<\boldsymbol{n}, \boldsymbol{m}, i, j>} \sum_{\sigma, \tau}\left\{V_{\mathrm{MoCu}} n_{n: \mathrm{Cu}(i) \sigma} n_{\boldsymbol{m}: \mathrm{Mo}(j) \tau}\right. \\
& +J_{\mathrm{MoCu}} c_{\boldsymbol{n}: \mathrm{Cu}(i) \sigma}^{\dagger} c_{m: \mathrm{Mo}(j) \tau}^{\dagger} c_{\boldsymbol{n}: \mathrm{Cu}(i) \tau} c_{\boldsymbol{m}: \mathrm{Mo}(j) \sigma} \\
& +\frac{J_{\mathrm{MoCu}}^{\prime}}{2}\left[c_{n: \mathrm{Cu}(i) \sigma}^{\dagger} c_{n: \mathrm{Cu}(i) \tau}^{\dagger} c_{m: \mathrm{Mo}(j) \tau} c_{m: \mathrm{Mo}(j) \sigma}+\text { H.c. }\right] \\
& \left.+\frac{t_{\mathrm{MoCu}}}{2}\left[(-1)^{i+j+1} c_{n: \mathrm{Cu}(i) \sigma}^{\dagger} c_{m: \mathrm{Mo}(j) \sigma}+\text { H.c. }\right]\right\} \\
& +\sum_{<\boldsymbol{n} \neq \boldsymbol{m}>} \sum_{i=1}^{4} \sum_{\sigma, \tau}\left\{V_{\mathrm{CuCu}} n_{\boldsymbol{n}: \mathrm{Cu}(i) \sigma} n_{\boldsymbol{m}: \mathrm{Cu}(\bar{i}) \tau}\right. \\
& +J_{\mathrm{CuCu}} c_{n: \mathrm{Cu}(i) \sigma}^{\dagger} c_{m: \mathrm{Cu}(\bar{i}) \tau}^{\dagger} c_{n: \mathrm{Cu}(i) \tau} c_{m: \mathrm{Cu}(\bar{i}) \sigma} \\
& +\frac{J_{\mathrm{CuCu}}^{\prime}}{2}\left[c_{n: \mathrm{Cu}(i) \sigma}^{\dagger} c_{n: \mathrm{Cu}(i) \tau}^{\dagger} c_{m: \mathrm{Cu}(\bar{i}) \tau} c_{m: \mathrm{Cu}(\bar{i}) \sigma}\right. \\
& \left.+ \text { H.c. }]+\frac{t_{\mathrm{CuCu}}}{2}\left[c_{n: \mathrm{Cu}(i) \sigma}^{\dagger} c_{m: \mathrm{Cu}(\bar{i}) \sigma}+\text { H.c. }\right]\right\},
\end{aligned}
$$

where $\bar{i}=i+4$. An electron number operator is defined as $n_{n: A(i)}=\sum_{\sigma} n_{n: A(i) \sigma}=\sum_{\sigma} c_{n: A(i) \sigma}^{\dagger} c_{n: A(i) \sigma}$, where $c_{n: A(i) \sigma}^{\dagger}$ creates the electron of spin $\sigma=\uparrow, \downarrow \equiv \pm$ on the $\mathrm{Cu} 3 d_{z x}$ $(A=\mathrm{Cu} ; i=1,2,5,6), \mathrm{Cu} 3 d_{y z}(A=\mathrm{Cu} ; i=3,4,7,8)$, or Mo $4 d_{z^{2}}(A=\mathrm{Mo} ; i=1,2,3,4)$ orbital at unit cell $\boldsymbol{n}$ [Fig.

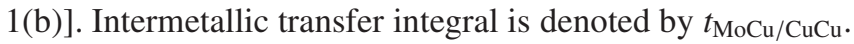
The on-site Coulomb repulsion $U_{\mathrm{Cu} / \mathrm{Mo}}$ and the orbital energy $\varepsilon_{\mathrm{Cu} / \mathrm{Mo}}$ of the isolated metals depend on their orbital radiuses. Intermetallic Coulomb, exchange, and pair-hopping interac- tions are denoted by $V_{\mathrm{MoCu} / \mathrm{CuCu}}, J_{\mathrm{MoCu} / \mathrm{CuCu}}$, and $J_{\mathrm{MoCu} / \mathrm{CuCu}}^{\prime}$, respectively. $\sum_{<\boldsymbol{n}, \boldsymbol{m}, i, j>}$ runs all Cu-Mo bonds, while $\sum_{<\boldsymbol{n} \neq \boldsymbol{m}>} \sum_{i=1}^{4}$ takes all pairs of the nearest neighboring $\mathrm{Cu}$ sites.

The model Hamiltonian on the $I 4 / m$ crystal structure has the point group symmetry $\mathbf{C}_{4 h} \equiv\left\{E, C_{4 z}^{-1}, C_{2 z}, C_{4 z}, \sigma_{h}, I C_{4 z}\right.$, $\left.I, I C_{4 z}^{-1}\right\}$. We investigate the photoinduced magnetic dynamics by solving the time-dependent Schrödinger equation, where the electron-electron interactions are treated within a HartreeFock (HF) scheme. Unless otherwise noted, the total number of units $N$ is set to $4096=16 \times 16 \times 16(\sim 50,000$ sites $)$. We confirmed that the static and dynamic properties of the larger $(N=13,824)$ system are qualitatively the same as those of the $N=4096$ system.
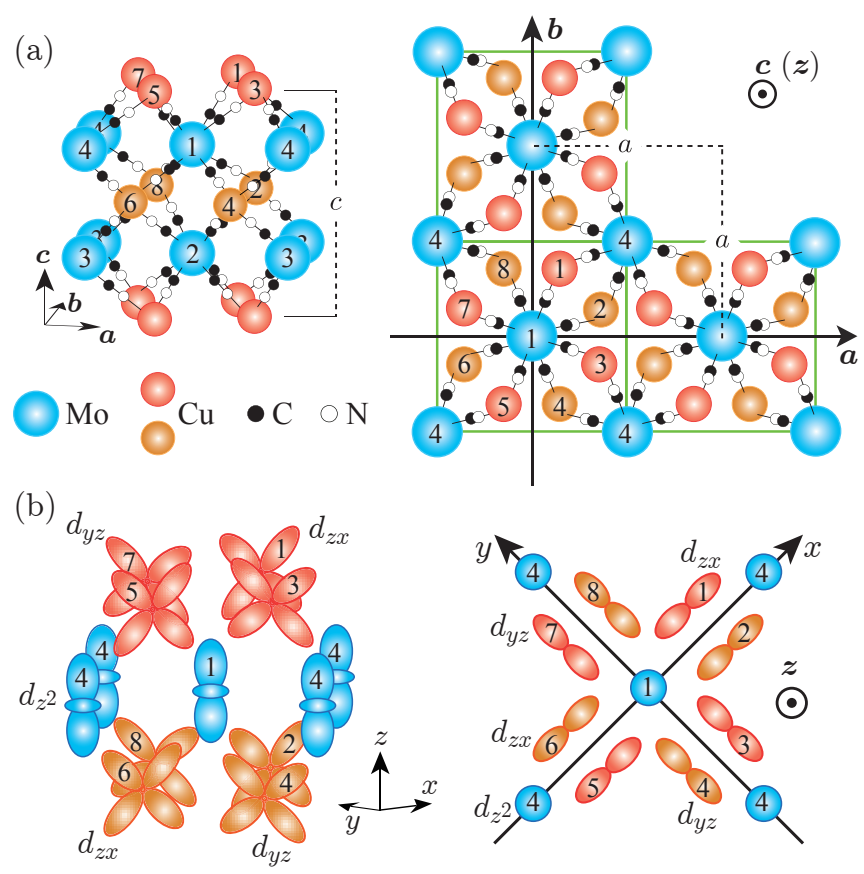

Fig. 1. (a) The $I 4 / m$ structure of the dicopper octacyanomolybdate, where the lattice constants $a$ and $c$ are set to $12 \AA .^{3)}$ (b) The arrangement of the Mo $d_{z^{2}}, \mathrm{Cu} 3 d_{z x}$, and $\mathrm{Cu} 3 d_{y z}$ orbitals. 


\section{S2. Competing ground states}

Figures 2(a) and 2(b) show possible electronic states of the dicopper octacyanomolybdate and their competition, respectively. The ground-state phase diagram of the Hubbard Hamiltonian $\mathcal{H}_{\mathrm{H}}$ [Fig. 2(b)] is obtained within the HF scheme, where the temperature $k_{\mathrm{B}} T / t_{\mathrm{MoCu}}$ is set to 0.001 in line with the experimental observation at the sufficiently low temperatures $3 \sim 10 \mathrm{~K}^{4)}$ The paramagnetic (PM) state having the full symmetry of the Hamiltonian can take the valence arrangement $\mathrm{Cu}^{\mathrm{II}}-\mathrm{Mo}^{\mathrm{IV}}-\mathrm{Cu}^{\mathrm{II}}$ and predominates in the moderate correlation regime. This state corresponds to the observed ground state. There exist two static magnetic solutions, namely ferromagnetic (FM) and spin-density-wave (SDW) states. The former shows a spatially uniform electron distribution, whereas the later exhibits inter-unit-cell antiferromagnetic ordering on $\mathrm{Mo}$ and $\mathrm{Cu}$ sublattices. In the strong correlation regime, such magnetic structures are generally stabilized as is discussed in other correlated electron systems. ${ }^{5-7)}$ However, the extremely strong $U_{\mathrm{Mo}}$ favors a nonmagnetic state. It is the chargedisproportionated $\left(\mathrm{CDP}\right.$ ) state taking $\mathrm{Cu}^{\mathrm{I}}-\mathrm{Mo}^{\mathrm{VI}}-\mathrm{Cu}^{\mathrm{I}}$ (fullyfilled $\mathrm{Cu}$ and vacant Mo sites). Given that the Mo $4 d$ orbital is more diffuse than the $\mathrm{Cu} 3 d$ one, the situation $U_{\mathrm{Mo}} \gg U_{\mathrm{Cu}}$ is not realistic and therefore the CDP state hardly appears as a ground state in the as-grown samples. In the weak correlation regime, the charge-density-wave (CDW) state is stabilized by the intermetallic Coulomb interaction $V_{\mathrm{CuCu}}$.

We focus on the PM state indicated by $\circ$ in Fig. 3(b) and investigate the photoexcitation mechanism. The model parameters are tuned so as to reproduce the $\mathrm{Cu}^{\mathrm{II}}-\mathrm{Mo}^{\mathrm{IV}}-\mathrm{Cu}^{\mathrm{II}}$ structure. This PM ground state shows a visible-light absorption [see the inset in Fig. 3(a) in the main text], which is in good agreement with the observations. ${ }^{4)}$ Since the PM state has an insulating gap of $0.4 \mathrm{eV}$ [see Fig. 5(b) in the main text], there is no thermal fluctuation effect at the present temperature. Electronic structures of the FM and CDP states are also discussed in the main text. These two states are indicated by symbols ( $\triangle$ and $\times)$ in the phase diagram.

\section{S3. Dzyaloshinskii-Moriya and Zeeman interactions}

A Dzyaloshinskii-Moriya (DM) interaction is given as

$$
\begin{aligned}
& \mathcal{H}_{\mathrm{DM}}=\sum_{n} \sum_{l=1}^{4} \sum_{\rho=0}^{1} \sum_{\sigma, \sigma^{\prime}=0}^{1}(-1)^{\rho+\sigma} \boldsymbol{D}_{l+4 \rho}^{\left(\sigma^{\prime} \sigma\right)} \\
& \cdot\left[\boldsymbol{s}_{\boldsymbol{n}+\delta\left(l, \rho, \sigma, \sigma^{\prime}\right): \operatorname{Mo}\left(1+3 \sigma^{\prime}+(-1)^{\sigma^{\prime}} \rho\right)} \times \boldsymbol{s}_{\boldsymbol{n}: \mathrm{Cu}(2 l-\sigma)}\right],
\end{aligned}
$$

where $\boldsymbol{\delta}\left(l, \rho, \sigma, \sigma^{\prime}\right)=\sigma^{\prime} \operatorname{Re}[f(l)](\boldsymbol{a} / a)+\sigma^{\prime} \operatorname{Im}[f(l)](\boldsymbol{b} / a)+$ $\sigma \rho(\boldsymbol{c} / c)$ with $f(l)=e^{\mathrm{i} \pi / 4}\left[1+\mathrm{e}^{\mathrm{i} \pi(1-l) / 2}\right] / \sqrt{2}$. An electron spin is given as $s_{n: A(i)}^{\lambda}=\sum_{\sigma, \sigma^{\prime}} c_{n: A(i) \sigma}^{\dagger} c_{n: A(i) \sigma^{\prime}} \tau_{\sigma \sigma^{\prime}}^{\lambda} / 2$ with the Pauli matrix $\tau^{\lambda}(\lambda=a, b, c)$. The DM vectors should be compatible with the crystalline structure as $\boldsymbol{D}_{i}^{\left(\sigma^{\prime} \sigma\right)}=g_{i} \cdot \boldsymbol{D}_{1}^{\left(\sigma^{\prime} \sigma\right)}$ with $g_{i}\left(\in \mathbf{C}_{4 h}\right)=C_{4 z}^{-1}, C_{2 z}, C_{4 z}, \sigma_{h}, I C_{4 z}, I, I C_{4 z}^{-1}$ for $i=2$ to 8 , respectively. We take $\boldsymbol{D}_{1}^{\left(\sigma^{\prime} \sigma\right)}=\boldsymbol{D}=D(\sin \theta, 0, \cos \theta)$ with $D=0.7 \mathrm{meV}$. The value of $D$ is comparable to that of typical transition-metal oxides. $\left.{ }^{8}{ }^{9}\right)$ When the DM vectors are parallel to the $c(z)$ direction $(\theta=0)$, the system is never photomagnetized because the $z$ component of $S_{A} \times S_{B}$ does not break the conservation law of the total magnetization: $\left[S_{\mathrm{A}} \times S_{\mathrm{B}}\right]_{z}=\left(S_{\mathrm{A}}^{-} S_{\mathrm{B}}^{+}-S_{\mathrm{A}}^{+} S_{\mathrm{B}}^{-}\right) / 4 i$. Once $\boldsymbol{D}$ has the $\boldsymbol{a}$ direction component, however, the system can be photomagnetized even though its in-plane component is very small. In the main text, the DM vectors lie on the $\boldsymbol{a}-\boldsymbol{b}$ plane $(\theta=\pi / 2)$.

We apply an external magnetic field along the $\boldsymbol{c}(\boldsymbol{z})$ axis to the system by employing the Zeeman Hamiltonian

$$
\mathcal{H}_{\mathrm{ex}}=-g \mu_{\mathrm{B}} H \sum_{n}\left[\sum_{i=1}^{8} s_{n: \mathrm{Cu}(i)}^{c}+\sum_{j=1}^{4} s_{n: \mathrm{Mo}(j)}^{c}\right],
$$

where $g \mu_{\mathrm{B}} H / t_{\mathrm{MoCu}}=2 \times 10^{-4}$. The degeneracy of up- and down-spin electrons is lifted, so that the down-spin electrons may selectively be photoexcited.

We confirmed that the DM and Zeeman interactions hardly change the phase diagram of $\mathcal{H}_{\mathrm{H}}$ [Fig. 2(b)] and have little effect on the static electronic structure of each state such as a dispersion relation.

\section{S4. Substitution of tungsten for molybdenum}

Let us consider substitution of $\mathrm{W}$ for Mo and its effects on the intermetallic transfer integral. When we apply a half-filled single-orbital Hubbard model to a two-site system consisting of $\mathrm{A}$ and $\mathrm{B}$ metals, the antiferromagnetic interaction

$$
J_{\mathrm{AB}}=t_{\mathrm{AB}}^{2}\left(\frac{1}{U_{\mathrm{A}}}+\frac{1}{U_{\mathrm{B}}}\right)
$$

is obtained in the strong-correlation limit, where $t_{\mathrm{AB}}$ is the AB transfer integral and $U_{\mathrm{A} / \mathrm{B}}$ is the on-site Coulomb repulsion. We here focus on the octacyanometalate-based $\mathrm{Mn}-\mathrm{Mo}^{10)}$ and Mn- $\mathrm{W}^{11)}$ antiferromagnets: $J_{\mathrm{MoMn}}=6.9 \mathrm{~cm}^{-1}$ and $J_{\mathrm{WMn}}=$ $4.6 \mathrm{~cm}^{-1}$. Substituting these parameters for Eq.(4), we obtain

$$
\frac{t_{\mathrm{WMn}}}{t_{\mathrm{MoMn}}}=\sqrt{0.66 \frac{U_{\mathrm{W}}+U_{\mathrm{Mn}}\left(U_{\mathrm{W}} / U_{\mathrm{Mo}}\right)}{U_{\mathrm{W}}+U_{\mathrm{Mn}}}} .
$$

In the situation $U_{\mathrm{Mn}(3 d)} \geq U_{\mathrm{Mo}(4 d)} \geq U_{\mathrm{W}(5 d)}, t_{\mathrm{WMn}} / t_{\mathrm{MoMn}}$ is less than unity. Assuming that $U_{\mathrm{Mo}} / U_{\mathrm{Mn}}=0.55$ and $U_{\mathrm{W}} / U_{\mathrm{Mo}}=0.60$, we obtain $t_{\mathrm{WMn}}=0.65 t_{\mathrm{MoMn}}$.

Based on the above consideration, we set $t_{\mathrm{WCu}}$ to $0.42 \mathrm{eV}$ $\left(=0.65 t_{\mathrm{MoCu}}\right)$ in the investigation of the tungsten analog. The other parameters are $t_{\mathrm{CuCu}}=0.3 t_{\mathrm{WCu}}, U_{\mathrm{W}}=2.0 t_{\mathrm{WCu}}$, $U_{\mathrm{Cu}}=3.2 t_{\mathrm{WCu}}, V_{\mathrm{CuCu}}=2.4 t_{\mathrm{WCu}}, V_{\mathrm{WCu}}=0.8 t_{\mathrm{WCu}}, \varepsilon_{\mathrm{Cu}}-\varepsilon_{\mathrm{W}}=$ $-1.0 t_{\mathrm{WCu}}$, and $J_{\mathrm{WCu}}=J_{\mathrm{WCu}}^{\prime}=-J_{\mathrm{CuCu}}=-J_{\mathrm{CuCu}}^{\prime}=0.6 t_{\mathrm{WCu}}$. These parameters are tuned so as to reproduce the $\mathrm{Cu}^{\mathrm{II}}-\mathrm{W}^{\mathrm{IV}}$ $\mathrm{Cu}^{\mathrm{II}}$ structure in the PM ground state. The obtained absorption spectrum is also consistent with the observations ${ }^{3)}$ [see the inset in Fig. 3(b) in the main text].

\section{S5. Polarized optical conductivity}

The regular part of the optical conductivity, $\sigma_{\lambda}^{\mathrm{reg}}(\omega)(\lambda=$ $a, b, c)$, is defined as

$$
\sigma_{\lambda}^{\mathrm{reg}}(\omega)=\frac{\pi}{N \omega} \sum_{i \neq 0}\left|\left\langle E_{i}\left|\mathcal{J}_{\lambda}\right| E_{0}\right\rangle\right|^{2} \delta\left(E_{i}-E_{0}-\hbar \omega\right),
$$



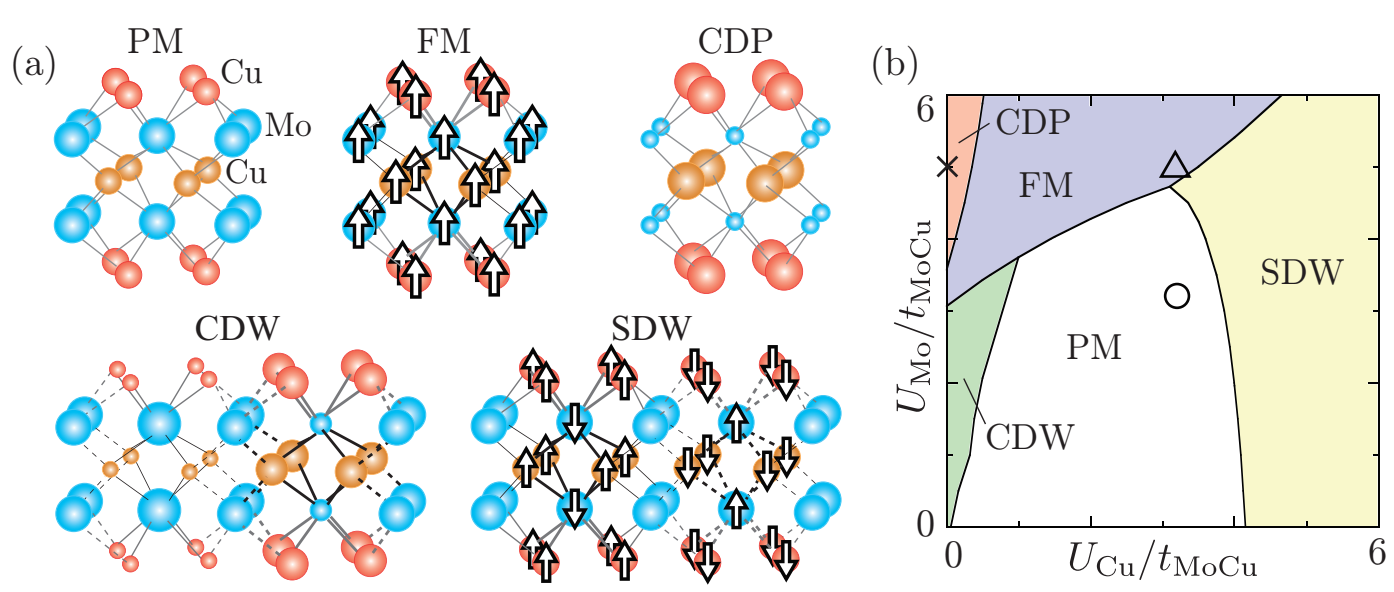

Fig. 2. (a) Schematic representations of possible mixed-valent states, where varying circles and arrows describe varying electron and spin densities, respectively. (b) A HF-ground-state phase diagram on the $U_{\mathrm{Cu}}-U_{\mathrm{Mo}}$ plane, where the symbols $\circ(3.2,3.2), \Delta(3.2,5.0)$, and $\times(0.0,5.0)$ indicate the PM, FM, and CDP states, respectively, in the investigation of photoinduced dynamics. The other parameters are given as follows, $t_{\mathrm{CuCu}}=0.3 t_{\mathrm{MoCu}}, V_{\mathrm{CuCu}}=2.4 t_{\mathrm{MoCu}}$, $V_{\mathrm{MoCu}}=0.8 t_{\mathrm{MoCu}}, \varepsilon_{\mathrm{Cu}}-\varepsilon_{\mathrm{Mo}}=1.0 t_{\mathrm{MoCu}}$, and $J_{\mathrm{MoCu}}=J_{\mathrm{MoCu}}^{\prime}=-J_{\mathrm{CuCu}}=-J_{\mathrm{CuCu}}^{\prime}=0.6 t_{\mathrm{MoCu}}$.

where a total unit number is denoted by $N$ and the current operator $\mathcal{J}_{\lambda}$ is given as

$$
\begin{aligned}
& \mathcal{J}_{\lambda}=\mathrm{i} \sum_{<\boldsymbol{n}, \boldsymbol{m}, i, j>} \sum_{\sigma} \frac{e r_{\mathrm{Cu}(i) ; M(j)}^{\lambda}}{\hbar}\left[(-1)^{i+j+1} t_{M \mathrm{Cu}}\right. \\
& -V_{M \mathrm{Cu}} p_{\boldsymbol{n}: \mathrm{Cu}(i) ; \boldsymbol{m}: M(j)}^{\sigma *}+J_{M \mathrm{Cu}} p_{\boldsymbol{n}: \mathrm{Cu}(i) ; \boldsymbol{m}: M(j)}^{*} \\
& \left.+J_{M \mathrm{Cu}}^{\prime} p_{\boldsymbol{n}: \mathrm{Cu}(i) ; \boldsymbol{m}: M(j)}^{-\sigma}\right] c_{n: \mathrm{Cu}(i) \sigma}^{\dagger} c_{\boldsymbol{m}: M(j) \sigma} \\
& +\mathrm{i} \sum_{<\boldsymbol{n} \neq \boldsymbol{m}>} \sum_{i=1}^{4} \sum_{\sigma} \frac{e r_{\mathrm{Cu}(i) ; \mathrm{Cu}(\bar{i})}^{\lambda}}{\hbar}\left[t_{\mathrm{CuCu}}-V_{\mathrm{CuCu}} p_{n: \mathrm{Cu}(i) ; \boldsymbol{m}: \mathrm{Cu}(\bar{i})}^{\sigma *}\right. \\
& \left.+J_{\mathrm{CuCu}} p_{n: \mathrm{Cu}(i) ; \boldsymbol{m}: \mathrm{Cu}(\bar{i})}^{*}+J_{\mathrm{CuCu}}^{\prime} p_{n: \mathrm{Cu}(i) ; \boldsymbol{m}: \mathrm{Cu}(\bar{i})}^{-\sigma}\right] \\
& \times c_{n: \mathrm{Cu}(i) \sigma}^{\dagger} c_{\boldsymbol{m}: \mathrm{Cu}(\bar{i}) \sigma}+\mathrm{H} . \mathrm{c.} . \quad(M=\mathrm{Mo}, \mathrm{W}) .
\end{aligned}
$$

A bond order is defined as $p_{\boldsymbol{n}: A(i) ; \boldsymbol{n}^{\prime}: A^{\prime}\left(i^{\prime}\right)}^{\sigma}=\left\langle c_{\boldsymbol{n}: A(i) \sigma}^{\dagger} c_{\boldsymbol{n}^{\prime}: A^{\prime}\left(i^{\prime}\right) \sigma}\right\rangle$, where $\langle\cdots\rangle$ means a canonical ensemble average, and a relative vector between $A(i)$ and $A^{\prime}\left(i^{\prime}\right)$ sites is denoted by $\boldsymbol{r}_{A(i) ; A^{\prime}\left(i^{\prime}\right)}=\left[r_{A(i) ; A^{\prime}\left(i^{\prime}\right)}^{a}, r_{A(i) ; A^{\prime}\left(i^{\prime}\right)}^{b}, r_{\left.A(i) ; A^{\prime}\left(i^{\prime}\right)\right]}^{c}\right]\left|E_{i}\right\rangle$ is an arbitrary wavefunction of energy $E_{i}\left(E_{0} \leq E_{1} \leq E_{2} \leq \cdots\right)$. The ground state $\left|E_{0}\right\rangle$ and the excited state $\left|E_{i}\right\rangle(i \neq 0)$ are given as

$$
\begin{aligned}
\left|E_{0}\right\rangle & =\prod_{\varepsilon_{\boldsymbol{k}, \chi} \leq \varepsilon_{\mathrm{F}}} \tilde{c}_{\boldsymbol{k}, \chi}^{\dagger}|0\rangle \quad(|0\rangle: \text { true electron vacuum }), \\
\left|E_{i}\right\rangle & =\tilde{c}_{\boldsymbol{k}, v}^{\dagger} \tilde{c}_{\boldsymbol{k}, \mu}\left|E_{0}\right\rangle \quad\left(\varepsilon_{\boldsymbol{k}, \mu} \leq \varepsilon_{\mathrm{F}}<\varepsilon_{\boldsymbol{k}, v}\right),
\end{aligned}
$$

respectively, where $\varepsilon_{\mathrm{F}}$ is the Fermi energy and $\tilde{c}_{\boldsymbol{k}, \chi}^{\dagger}$ creates an electron of the HF energy $\varepsilon_{k, \chi}(\chi=1 \sim 24)$.

The Drude component of the optical conductivity, $\sigma_{\lambda}^{\mathrm{D}}$, is defined as

$$
\sigma_{\lambda}^{\mathrm{D}}=-\frac{\pi}{N}\left(\left\langle E_{0}\left|\mathcal{T}_{\lambda}\right| E_{0}\right\rangle+2 \sum_{i \neq 0} \frac{\left|\left\langle E_{i}\left|\mathcal{J}_{\lambda}\right| E_{0}\right\rangle\right|^{2}}{E_{i}-E_{0}}\right)
$$

where the kinetic-energy operator $\mathcal{T}_{\lambda}$ is given as

$$
\begin{aligned}
& \mathcal{T}_{\lambda}=\sum_{<\boldsymbol{n}, \boldsymbol{m}, i, j>} \sum_{\sigma}\left[\frac{e r_{\mathrm{Cu}(i) ; M(j)}^{\lambda}}{\hbar}\right]^{2}\left[(-1)^{i+j+1} t_{M \mathrm{Cu}}\right. \\
& -V_{M \mathrm{Cu}} p_{n: \mathrm{Cu}(i) ; m: M(j)}^{\sigma *}+J_{M \mathrm{Cu}} p_{n: \mathrm{Cu}(i) ; m: M(j)}^{*} \\
& \left.+J_{M \mathrm{Cu}}^{\prime} p_{\boldsymbol{n}: \mathrm{Cu}(i) ; \boldsymbol{m}: M(j)}^{-\sigma}\right] c_{\boldsymbol{n}: \mathrm{Cu}(i) \sigma}^{\dagger} c_{\boldsymbol{m}: M(j) \sigma} \\
& +\sum_{<\boldsymbol{n} \neq \boldsymbol{m}>} \sum_{i=1}^{4} \sum_{\sigma}\left[\frac{e r_{\mathrm{Cu}(i) ; \mathrm{Cu}(\bar{i})}^{\lambda}}{\hbar}\right]^{2}\left[t_{\mathrm{CuCu}}-V_{\mathrm{CuCu}} p_{n: \mathrm{Cu}(i) ; \boldsymbol{m}: \mathrm{Cu}(\bar{i})}^{\sigma *}\right. \\
& \left.+J_{\mathrm{CuCu}} p_{\boldsymbol{n}: \mathrm{Cu}(i) ; \boldsymbol{m}: \mathrm{Cu}(\bar{i})}^{*}+J_{\mathrm{CuCu}}^{\prime} p_{\boldsymbol{n}: \mathrm{Cu}(i) ; \boldsymbol{m}: \mathrm{Cu}(\bar{i})}^{-\sigma}\right] \\
& \times c_{n: \mathrm{Cu}(i) \sigma}^{\dagger} c_{m: \mathrm{Cu}(\bar{i}) \sigma}+\text { H.c. } \quad(M=\mathrm{Mo}, \mathrm{W}) .
\end{aligned}
$$

The Drude weight is closely related to the kinetic energy of electrons. The total optical conductivity is given as

$$
\sigma_{\lambda}(\omega)=\sigma_{\lambda}^{\mathrm{reg}}(\omega)+\sigma_{\lambda}^{\mathrm{D}} \delta(\omega)
$$

In the time-evolutional calculation, the initial state $\left|E_{0}\right\rangle$ is replaced by the time-evolutional wavefunction $|\Psi(t)\rangle$. The one-electron-hole pair excited state from $|\Psi(t)\rangle$ is defined as

$$
\left|E_{i}(t)\right\rangle=\tilde{c}_{\boldsymbol{k}, v}^{\dagger} \tilde{c}_{\boldsymbol{k}, \mu}|\Psi(t)\rangle,
$$

where $\tilde{c}_{\boldsymbol{k}, v}^{\dagger}\left(\tilde{c}_{\boldsymbol{k}, \mu}\right)$ creates (destroys) an electron of the HF-level energy of $\mathcal{H}_{\mathrm{HF}}(t)$. The regular part and Drude component of the optical conductivity are given as

$$
\sigma_{\lambda}^{\mathrm{reg}}(t ; \omega)=\frac{\pi}{N \omega} \sum_{i \neq 0}\left|\left\langle E_{i}(t)\left|\mathcal{J}_{\lambda}\right| \Psi(t)\right\rangle\right|^{2} \delta\left(\left\langle E_{i}\right\rangle-\left\langle E_{0}\right\rangle-\hbar \omega\right)
$$

and

$$
\sigma_{\lambda}^{\mathrm{D}}(t)=-\frac{\pi}{N}\left(\left\langle\Psi(t)\left|\mathcal{T}_{\lambda}\right| \Psi(t)\right\rangle+2 \sum_{i \neq 0} \frac{\left|\left\langle E_{i}(t)\left|\mathcal{J}_{\lambda}\right| \Psi(t)\right\rangle\right|^{2}}{\left\langle E_{i}\right\rangle-\left\langle E_{0}\right\rangle}\right),
$$


respectively, where

$$
\begin{aligned}
\left\langle E_{0}\right\rangle & =\left\langle\Psi(t)\left|\mathcal{H}_{\mathrm{HF}}(t)\right| \Psi(t)\right\rangle, \\
\left\langle E_{i}\right\rangle & =\left\langle E_{i}(t)\left|\mathcal{H}_{\mathrm{HF}}(t)\right| E_{i}(t)\right\rangle .
\end{aligned}
$$

This is the naivest extension of Eqs. (6) and (10). A formulation of dynamical correlation functions in nonequilibrium states is challenging topic and there are several researches. One of them treats both pump and probe photons explicitly and considers inter-excited-state transitions. ${ }^{12)}$ We confirmed that the visible absorption spectra obtained with this method are qualitatively the same as those by Eqs. (14) and (15).

\section{S6. Parameter dependences of the photoinduced magneti- zation dynamics}

In the time-evolutional calculation, we define the numbers of excited up-spin $(\uparrow)$ and down-spin $(\downarrow)$ electrons per site,

$$
\begin{aligned}
& n_{\mathrm{ex}}^{(\uparrow)}=\frac{1}{12 N} \sum_{k} \sum_{\chi=17}^{20}\left\langle\Psi(t)\left|\tilde{c}_{\boldsymbol{k}, \chi}^{\dagger} \tilde{c}_{\boldsymbol{k}, \chi}\right| \Psi(t)\right\rangle, \\
& n_{\mathrm{ex}}^{(\downarrow)}=\frac{1}{12 N} \sum_{\boldsymbol{k}} \sum_{\chi=21}^{24}\left\langle\Psi(t)\left|\tilde{c}_{\boldsymbol{k}, \chi}^{\dagger} \tilde{c}_{\boldsymbol{k}, \chi}\right| \Psi(t)\right\rangle .
\end{aligned}
$$

Figure 3 shows $n_{\mathrm{ex}}^{(\uparrow)}, n_{\mathrm{ex}}^{(\downarrow)}$, and $\Delta n\left(=n_{\mathrm{ex}}^{(\downarrow)}-n_{\mathrm{ex}}^{(\uparrow)}\right)$ as functions of time. Since the spin degeneracy is lifted due to the external magnetic field, the higher-lying down-spin electrons are selectively excited by the first photoirradiation. This spinselective electron transfer induces a net spin density on each site and then the DM interaction begins to work. When the system is further irradiated with the longer-wavelength lights, $\Delta n$ gets smaller.

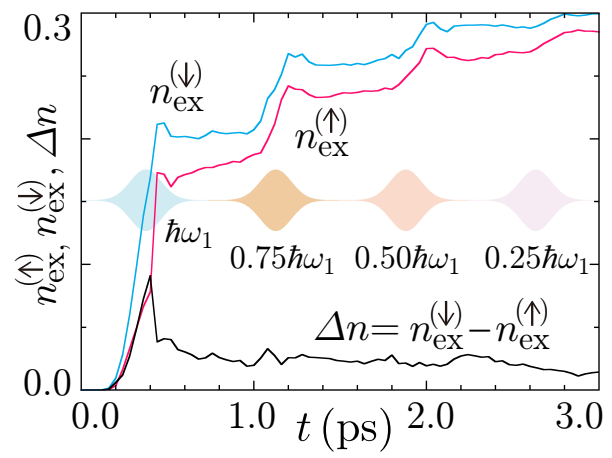

Fig. 3. The numbers of excited up-spin $(\uparrow)$ and down-spin $(\downarrow)$ electrons per site in the magnetic phase transition. The model parameters are the same as those in Fig. 4(a) in the main text.

Figure 4(a) shows the magnetic-field $(H)$ dependence of the induced magnetization. A change in $H$ has little effect on the magnetization dynamics. In contrast, the magnetization dynamics strongly depends on the laser amplitude $A$ as is shown in Fig. 4(b). When the magnetization rises at time $t_{0}$ and reaches its maximum value $M_{\max }$ at time $t_{1}$, the switching speed is defined as

$$
v_{\mathrm{s}}=\frac{M_{\mathrm{max}} / M_{\mathrm{sat}}}{t_{1}-t_{0}},
$$

where $M_{\text {sat }}$ means the saturation magnetization. The absorption energy $\Delta E$ is given by

$$
\Delta E=\left\langle\Psi(t)\left|\mathcal{H}_{\mathrm{HF}}(t)\right| \Psi(t)\right\rangle-\left\langle\Psi(-\infty)\left|\mathcal{H}_{\mathrm{HF}}(t)\right| \Psi(-\infty)\right\rangle .
$$

In the moderate excitation regime, $\Delta E$ is proportional to the laser power $A^{2}$, whereas $v_{\mathrm{s}}$ is proportional to the laser amplitude $A$, as is shown in Fig. 5. The amplitude $A$ is decisive for the time scale of the magnetization dynamics.

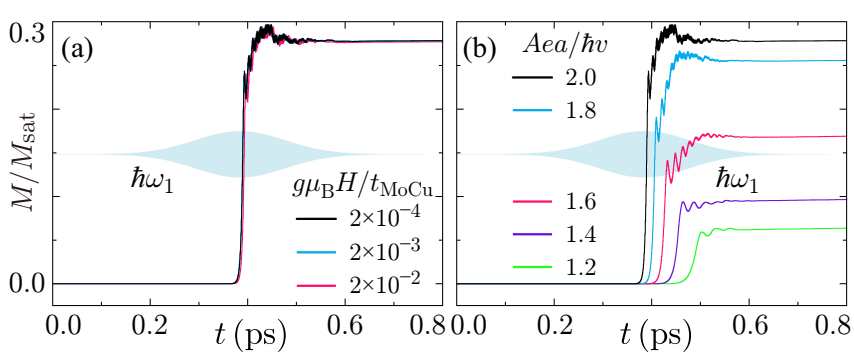

Fig. 4. (a) $H$ and (b) $A$ dependences of the magnetization dynamics. The other parameters are the same as those in Fig. 4(a) in the main text. The black lines correspond to the result presented in the main text.
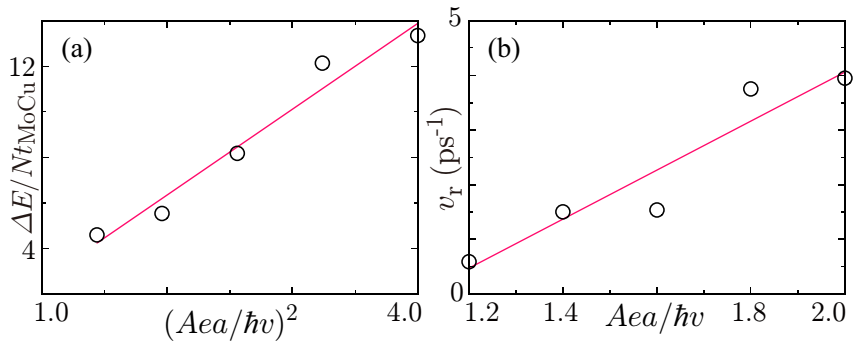

Fig. 5. (a) The absorption energy $\Delta E$ at $t=0.8 \mathrm{ps}$ as a function of the laser power $A^{2}$. (b) The switching speed $v_{\mathrm{s}}$ as a function of the laser amplitude $A$. Lines are guides to eyes.

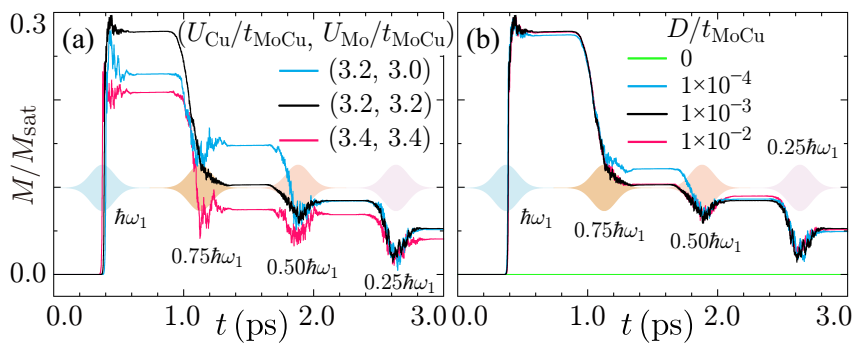

Fig. 6. The same as Fig. 4(a) in the main text, but for different (a) $U_{\mathrm{Cu} / \mathrm{Mo}}$ and (b) $D$. The black lines correspond to the result presented in the main text. 
Figure 6(a) shows the initial-state dependences of the photoinduced phase transition, where the on-site Coulomb repulsions are changed within the PM region. The induced magnetization is quantitatively different depending on the electronic correlations but the photoswitching of magnetization is observed in all cases. Thus, the photoinduced multistep phase transition occurs at around the present parameter point. We further present the effects of the DM interaction in Fig. 6(b). When $D=0$, no magnetization appears because of the conservation law of the magnetization. Once $D$ has a finite value, however, the system can be photomagnetized even though its value is very small. The magnetization and demagnetization dynamics hardly depend on the magnitude of $D$.

1) J. E. Hirsch, Phys. Rev. B 43, 705 (1991).
2) R. Strack and D. Vollhardt, J. Low Temp. Phys. 99, 385 (1995).

3) G. Rombaut, C. Mathonière, P. Guionneau, S. Golhen, L. Ouahab, M. Verelst, and P. Lecante, Inorg. Chim. Acta 326, 27 (2001).

4) S. Ohkoshi, H. Tokoro, T. Hozumi, Y. Zhang, K. Hashimoto, C. Mathonière, I. Bord, G. Rombaut, M. Verelst, C. C. dit Moulin, and F. Villain, J. Am. Chem. Soc. 128, 270 (2006).

5) J. E. Hirsch, Phys. Rev. B 40, 2354 (1989).

6) J. Wahle, N. Blümer, J. Schlipf, K. Held, and D. Vollhardt, Phys. Rev. B 58, 12749 (1998).

7) M. J. Calderón, G. León, B. Valenzuela, and E. Bascones, Phys. Rev. B 86, 104514 (2012).

8) J. Stein, O. Entin-Wohlman, and Amnon Aharony, Phys. Rev. B 53, 775 (1996).

9) B. Keimer, D. Casa, A. Ivanov, J. W. Lynn, M. v. Zimmermann, J. P. Hill, D. Gibbs, Y. Taguchi, and Y. Tokura, Phys. Rev. Lett. 85, 3946 (2000).

10) Q.-L. Wang, Y.-Z. Zhang, H. Southerland, A. V. Prosvirin, H. Zhao, and K. R. Dunbar, Dalton Trans. 43, 3802 (2014).

11) C. Yang, Q.-L. Wang, C.-Y. Su, L.-N. Hu, L.-C. Li, and D.-Z. Liao, Inorg. Chem. Commun. 40, 26 (2014).

12) J. Ohara, Y. Kanamori, and S. Ishihara, Phys. Rev. B 88, 085107 (2013). 